\title{
3D Multi-fluid MHD Studies of the Solar Wind Interaction with Mars
}

\author{
Yifan Liu, Andrew F. Nagy, Clinton P. T. Groth, \\ Darren L. DeZeeuw, Tamas I. Gombosi \\ Space Physics Research Laboratory, Department of Atmospheric, Oceanic and Space Sciences, \\ University of Michigan, Ann Arbor, MI, 48109
}

Kenneth G. Powell

Aerospace Engineering Department, University of Michigan, Ann Arbor, MI, 48109

\begin{abstract}
The interaction of the solar wind with planets with no or only very weak intrinsic magnetic fields, such as Mars and Venus, involves their ionospheres. Single fluid MHD models, which incorporate some of the important mass-loading processes, have been useful in reproducing numerous observed features at these planets, such as the location of the bow shock, but they do have obvious limitations. Our present 3D MHD model is a two-fluid one, which considers protons and the dominant heavy ions in the ionosphere, separately. We have used this model to study the interaction processes at Mars. The model results are in general agreement with the average observed bow shock shape and position and predict reasonable locations for the ionopause. The calculated oxygen escape flux down the tail was estimated to be $2.7 \times 10^{25} \mathrm{~s}^{-1}$, which is consistent with Phobos-2 estimates.
\end{abstract}

\section{Introduction}

The magnetometer carried aboard the Mars Global Surveyor (MGS) has clearly demonstrated that, like Venus, Mars does not have a significant, global, intrinsic magnetic field [Acuña et al., 1998]. These measurements also established that at Mars, unlike Venus, some local, small-scale, crustal, remnant magnetization is present [Acuña et al., 1998]. Therefore, the global scale solar wind interaction with Mars is with the ionosphere/atmosphere system, directly. Studies of the solar wind interaction with unmagnetized bodies (e.g. Venus, Mars, Titan) have been carried out in the past using both semi-kinetic (e.g. Brecht [1997]) and single-fluid, ideal MHD model calculations (e.g. $\mathrm{Mu}$ rawski and Steinolfson [1996]; Bauske et al. [1998]; Shinagawa and Bougher [1999]). The single fluid MHD calculations have, in general, considered the planet/moon as a perfectly conducting sphere. These model studies have been successful in predicting numerous observed features, such as the bow shock position. Semi-kinetic calculations of the solar wind interaction with Venus

Copyright 1999 by the American Geophysical Union.

Paper number 1999GL900584.

0094-8276/99/1999GL900584\$05.00 and Mars have also used conducting spheres as inner boundary conditions. These model calculations have been especially useful in showing the lack of symmetry in the interaction processes. It has been argued that the use of semi-kinetic models is especially appropriate for Mars, where the ion gyroradius is of the same order as the planetary radius. However, it is important to note that the ideal MHD equations have been found to be successful even in such situations. The possible reason for this may be the fact that, as shown by the semi-kinetic code, and observed by the Phobos- 2 wave instrument [Grard et al., 1989], significant wave activity is present, leading to a wide variety of wave particle interactions, which in turn act as pseudo-collisions.

A two-fluid $\left(\mathrm{H}^{+}\right.$and $\left.\mathrm{O}^{+}\right)$ideal MHD model, to study the interaction of the solar wind with Venus was developed recently (Tanaka and Murawski [1997]; Tanaka [1998]), which for the first time used an actual, realistic ionosphere as the obstacle to the solar wind. Their results for nominal ionospheric conditions are in general agreement with the Pioneer Venus data base.

\section{Model and numerical method}

In this paper we present the first results from our two-fluid ideal MHD calculations of the solar wind interaction with Mars. We solve the ideal coupled MHD equation set, consisting of two continuity, one momentum, one energy and the magnetic induction equations. The source vector, $\mathbf{Q}$, used is:

$$
\mathbf{Q}=\left(\begin{array}{c}
0 \\
S_{2}-L_{2} \\
0 \\
\left(\rho_{1}+\rho_{2}\right) \mathbf{g}-\left(\rho_{1}+\rho_{2}\right) \nu \mathbf{u}-\mathbf{u} L_{2} \\
\left(\rho_{1}+\rho_{2}\right) \mathbf{u} \cdot \mathbf{g}-\frac{1}{2} u^{2} L_{2}-\left(\rho_{1}+\rho_{2}\right) \nu u^{2} \\
-\frac{1}{\gamma-1} \frac{L_{2} p}{32 \rho_{1}+\rho_{2}}+\frac{1}{\gamma-1} S_{2} \frac{k}{m_{2}} T_{0}
\end{array}\right)
$$

and where $\rho_{1}$ and $\rho_{2}$ are the $\mathrm{H}^{+}$and $\mathrm{O}_{2}^{+}$mass densities, respectively, $S_{2}$ and $L_{2}$ are the $\mathrm{O}_{2}^{+}$mass source and loss rates, respectively, $p$ is the total thermal pressure of the plasma, $\mathbf{u}$ is the velocity of the plasma, $\nu$ is the ion neutral collision frequency (taken to be $\left.4 \times 10^{-10}\left\{[\mathrm{O}]+\left[\mathrm{CO}_{2}\right]\right\} s^{-1}\right), T_{0}$ is the temperature of the 
newly produced $\mathrm{O}_{2}^{+}$and the other symbols have their usual definition.

The Viking 1 and 2 retarding potential analyzer (RPA) measurements [Hanson et al., 1977] and subsequent theoretical models (Chen et al. [1978]; Fox [1993]) have shown that $\mathrm{O}_{2}^{+}$is the major dayside ion below about $300 \mathrm{~km}$ in the ionosphere of Mars. Thus, in contrast to Venus, we selected $\mathrm{H}^{+}$and $\mathrm{O}_{2}^{+}$as the two ions to use in our two fluid model. The chemical reactions that we considered for the production and loss of $\mathrm{O}_{2}^{+}$ions are:

$$
\begin{gathered}
\mathrm{CO}_{2}+h \nu \rightarrow \mathrm{CO}_{2}^{+}+e \\
\mathrm{CO}_{2}^{+}+\mathrm{O} \rightarrow \mathrm{O}_{2}^{+}+\mathrm{CO} \\
\mathrm{O}_{2}^{+}+e \rightarrow \mathrm{O}+\mathrm{O}
\end{gathered}
$$

We made the assumption that $[e] \approx\left[\mathrm{O}_{2}^{+}\right]$and thus the source of $\mathrm{O}_{2}^{+}$is approximately $\mathrm{I}_{\mathrm{CO}_{2}}\left[\mathrm{CO}_{2}\right] \cos (S Z A)$ where SZA is the solar zenith angle, where $I_{\mathrm{CO}_{2}}$ is the photoionization rate of $\mathrm{CO}_{2}$ (taken to be $7.3 \times 10^{-7} \mathrm{~s}^{-1}$ [Schunk and Nagy, 1999]). The loss rate is $k_{4}\left[\mathrm{O}_{2}{ }^{+}\right]^{2}$, where $k_{4}$ is the dissociative recombination rate (taken to be $7.38 \times 10^{-8} \mathrm{~cm}^{3} \mathrm{~s}^{-1}$ [Schunk and Nagy, 1999]). These assumptions admittedly oversimplify the ionospheric photochemistry, but do provide a first order approximation for the $\mathrm{O}_{2}^{+}$density.

A modified version of the BATS-R-US (Block Adaptive Tree Solar-wind Roe-type Upwind Scheme) has been developed to solve the two-fluid MHD equations described above. The BATS-R-US solution method is a highly scalable, massively parallel, block-adaptive mesh refinement algorithm developed for space physics applications that uses recent algorithmic advances in highresolution upwind schemes. Details of the approach are described elsewhere. Refer to the papers by Powell et al. [1999], Groth et al. [1999], and DeZeeuw et al. [1999] for details. The basic elements of the modified solver are: 1) a finite-volume approach that solves the fluid and electromagnetic equations in a tightly coupled manner; 2) a flux function based on a new 9-wave approximate Riemann solver; 3) limited linear reconstruction that provides second-order accuracy away from high-gradient regions and monotonic nonoscillatory solutions; 4) explicit multi-stage time stepping with source terms treated point-implicitly; 5) blockbased solution-adaptive Cartesian mesh with physicsbased refinement; and 6) a parallel implementation that provides high performance on a variety of parallel architectures. The resulting parallel adaptive solution algorithm is both robust and accurate across a wide range of values of plasma conditions.

A computational domain defined by $-24 R_{M} \leq x \leq$ $8 R_{M},-16 R_{M} \leq y, z \leq 16 R_{M}$, where $R_{M}=3396 \mathrm{~km}$ is the radius of Mars, was used in the calculations and the inner boundary was taken to be $140 \mathrm{~km}$ above the Martian surface. An adapted grid consisting of 16,612 blocks and 1,063,168 computational cells was used in all of the simulations with the smallest cells having a dimension of about $53 \mathrm{~km}$ near the inner boundary and the largest cells, located in the nightside regions far from the planet, having a length of about $6792 \mathrm{~km}$. The inner boundary conditions were specified as follows: $\rho_{1}=0.3 \rho_{s w}$, where $\rho_{s w}$ is the mass density of the undisturbed solar wind. This boundary condition is consistent with the expectation of very low $\mathrm{H}^{+}$densities in the lower ionosphere, similar to the situation at Venus (e.g. [Taylor et al., 1980]). The $\mathrm{O}_{2}^{+}$density at the inner boundary was taken to be the photochemical equilibrium value. $B$ was set equal to zero and a reflective boundary was used for $\mathbf{u}$. This boundary condition for $\mathbf{u}$, results in near zero velocities at the inner boundary and ionospheric velocities of a few $k m s^{-1}$ in the ionosphere, as expected, assuming Venus-like conditions (c.f. [Miller and Whitten, 1991]). The sum of the electron and ion temperatures at the inner boundary was assumed to be $3000^{\circ} \mathrm{K}$ and the pressure was set accordingly. The upstream solar wind ion and electron temperatures were set to be $5 \times 10^{4}$ and $3 \times 10^{5}{ }^{\circ} \mathrm{K}$ respectively. The IMF was assumed to be a Parker spiral in the X-Y plane with an angle of 56 degrees and a magnitude of $3 \mathrm{nT}$ and the solar wind density and velocity were selected to be $4 \mathrm{~cm}^{-3}$ and $500 \mathrm{~km} \mathrm{~s}^{-1}$, respectively.

\section{Results}

The extensive computer resources necessary to run this model limits the number of cases that we are able to run. We used the NASA/GSFC Cray T3E parallel computer, running on 256 processors; each run required about 1000 processor hours. In Figure 1 (upper panel) we plot various calculated parameters along the subsolar line, for the nominal case indicated in the previous section. We show the variations of the kinetic
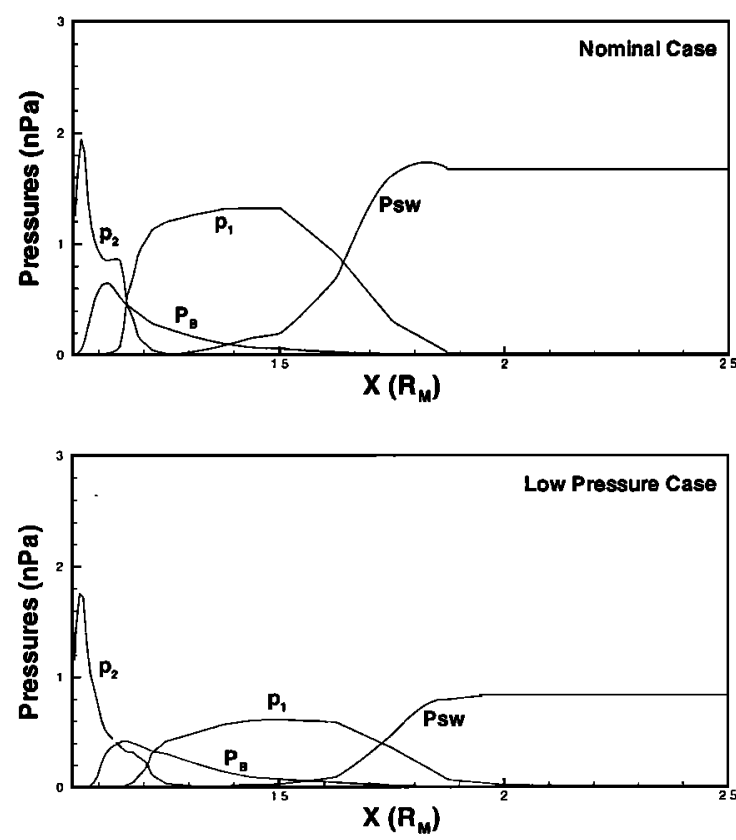

Figure 1. The distributions of kinetic pressure of the solar wind $\left(P_{s w}\right)$, thermal pressure of proton $\left(p_{1}\right)$ and that of $\mathrm{O}_{2}^{+}\left(p_{2}\right)$, and magnetic pressure $\left(P_{B}\right)$ along the Sun-Mars line on the dayside for the nominal case (upper panel) and the low pressure case (lower panel). 

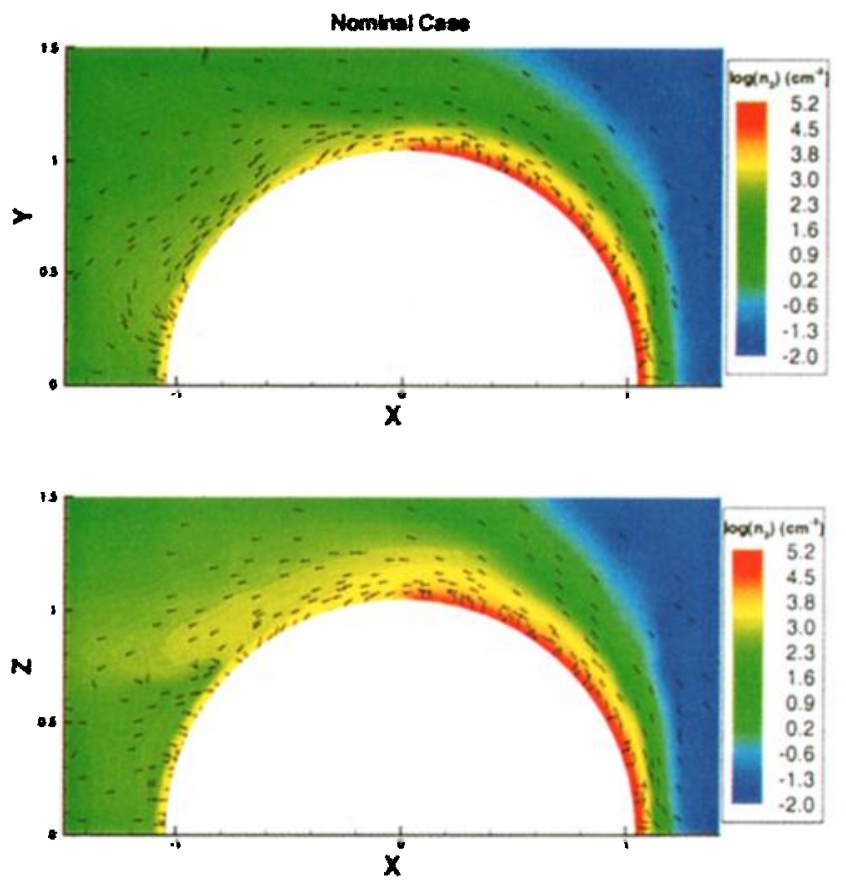

Figure 2. Contour plots of $\log \left(n_{2}\right)$ (where $n_{2}$ is the number density of $\mathrm{O}_{2}^{+}$in unit of $\mathrm{cm}^{-3}$ ) in X-Y (upper panel) and X-Z plane (lower panel) for the nominal case. The arrows show the direction of $\mathrm{O}_{2}^{+}$flux vector in the corresponding plane.

(ram) pressure of the solar wind, the thermal pressures of proton and molecular oxygen ions, and the magnetic pressure along the Sun-Mars line. A clear ionopause is seen at approximately $1.16 R_{M}$, which corresponds to an altitude of about $543 \mathrm{~km}$. The subsolar and terminator bow shocks are located at around 1.64 and 2.7 $R_{M}$, respectively. These calculated shock positions are in very good agreement with the observed MGS values [Vignes et al., 1999]. We need to note that in the bow shock region the spatial resolution in our model is only about $0.125 R_{M}$, necessitated by computer resources. This coarse spatial resolution is the cause of the indicated width of the shock. The formation of a magnetic barrier is also clearly visible. Along the subsolar line the peak of the magnetic field magnitude is about $\mathbf{4 0}$ $\mathrm{nT}$, just inside the ionopause, at a stand-off distance of about $1.11 R_{M}$. It is difficult to compare the calculated location of the magnetic barrier with the measurements, because of certain ambiguities in the way the observed results are presented. The MGS observations given by Vignes et al. [1999] put the subsolar magnetic pile up boundary, MPB, at about $1.41 R_{M}$, further out than our calculated location of the peak magnetic value. However, the magnetic barrier is a relatively wide structure and the choice of the MPB location in that study is not defined in terms of the maximum in the total field (the choice is based on a combination of electron flux, magnetic field magnitude and fluctuation changes). Trotignon et al. [1996] summarize the observed "planetopause" locations, with a subsolar position of $1.19 R_{M}$, closer to our calculated value, but the relation between MPB and the planetopause has not been clearly established.

We also ran a low solar wind pressure case, by reducing the solar wind density to $2 \mathrm{~cm}^{-3}$, while keeping the velocity unchanged. The subsolar results for this case are shown in Figure 1 (lower panel). The subsolar ionopause and bowshock locations are at about 1.2 and $1.73 R_{M}$, respectively, for this low pressure case. The bow shock location moved only slightly (about one grid point) when the solar wind pressure was decreased, similar to the situation found at Venus [Slavin et al., 1980]. On the other hand the ionopause location, on a relative altitude scale, moved more noticeably outward, to $680 \mathrm{~km}$, also consistent with observations at Venus (e.g. Brace et al. [1980]). The large data base of radio occultation electron density profiles obtained by Mariner and the Viking spacecraft do not show any clear ionopause signatures up to about $300 \mathrm{~km}$, their effective range of reliable observations. The MGS reflectrometer low energy electron measurements place the mean ionopause at $350 \mathrm{~km}$, during medium solar activity conditions [D. Mitchell, personal communication]. Our calculations correspond to solar maximum conditions and thus our calculated higher ionopause location is not unreasonable.

Figure 2 shows the contour plots of $\log \left(n_{2}\right)$ in the X$\mathrm{Y}$ plane (upper panel) and the X-Z plane (lower panel) calculated for the nominal case. The peak dayside densities are just below $2 \times 10^{5} \mathrm{~cm}^{-3}$, consistent with the observed and model values (Hanson et al. [1977]; Chen et al. [1978]; Fox [1993]). The calculated nightside ion densities are of the order of a few times $10^{3} \mathrm{~cm}^{-3}$, consistent with the observed values [Zhang et al., 1990]. The arrows in Figure 2 show the direction of the $\mathrm{O}_{2}^{+}$ flux vector $\left(n_{2} \mathbf{u}\right)$. At the dayside, the flow in the ionosphere is generally upward. At increasing zenith an-

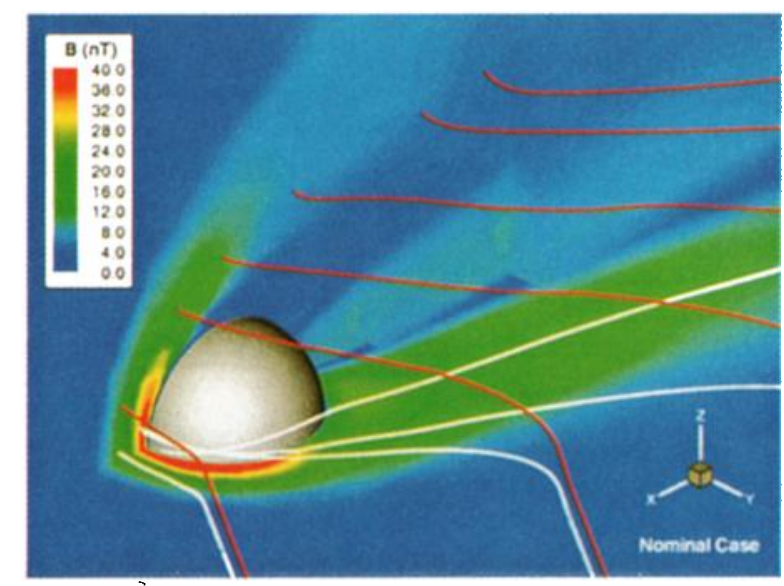

Figure 3. Three dimensional configuration of magnetic field for the nominal case. Contour plots show the magnetic field magnitude $(B)$ in equatorial plane (horizontal plane) and meridian plane (vertical plane). The white and red lines are the magnetic field lines with starting points at 0.1 and $0.5 R_{M}$ above the equatorial plane, respectively. 
gles the topside ionospheric flow drapes poleward and nightward, which is followed by downward (towards the planet) flow in the near tail region. The stagnation point along the Sun-Mars line in the central tail is located at about $1.5 R_{M}$. Beyond this region the flow is tailward. The total $\mathrm{O}_{2}^{+}$flux flowing down the tail and escaping from the planet is approximately $2.7 \times 10^{25} \mathrm{~s}^{-1}$, which compares favorably with the estimated value of $3 \times 10^{25} s^{-1}$, based on local flux measurements by the ASPERA instrument carried aboard Phobos 2 [Lundin et al., 1989]. In our model the only ionospheric species that we considered is $\mathrm{O}_{2}^{+}$, but in reality the escape flux must be composed of both $\mathrm{O}_{2}^{+}$and $\mathrm{O}^{+}$ions.

Figure 3 shows contour plots of the magnitude of $\mathbf{B}$ and certain draped field lines. The white and red lines indicate field lines which started out, in front of the planet, at 0.1 and $0.5 R_{M}$ above the equatorial plane, respectively. Our simulation results produce an induced magnetotail like that of Venus, consisting of two basically symmetric lobes (the Parker angle introduces a small asymmetry). The features of the magnetotail are controlled by the orientation of the IMF.

\section{Conclusion}

Here we presented the first results of our two fluid MHD model of the interaction of the solar wind with Mars. The two ions considered are $\mathrm{H}^{+}$and $\mathrm{O}_{2}^{+}$, representing the solar wind and the major ionospheric ion species, respectively. The results have been compared with observed parameters, which have been obtained by the Mars Global Surveyor and Phobos-2 missions. The general agreement between the model and observed values is quite good; further calculations with different solar wind and ionospheric parameters are planned. Also, we plan to introduce changes to the model, which will allow better altitude resolution, while reducing the needed computer resources.

Acknowledgments. This work was supported by NASA grants NAG5-4912 and HPCC CAN NCCS5-146; additional computing resources on the GSFC HPCC Cray T3E (JSIMPSON) were also received and are acknowledged.

\section{References}

Acuña, M. H., et al., Magnetic field and plasma observations at Mars: Initial results of the Mars Global Surveyor mission, Science, 279, 1676, 1998.

Bauske, R., et al., A three-dimensional MHD study of solar wind mass loading processes at Venus: Effects of photoionization, electron impact ionization, and charge exchange, J. Geophys. Res., 103, 23,625, 1998.

Brace, L. H., et al., The dynamic behavior of the Venus ionosphere in response to solar wind interactions, J. Geophys. Res., 85, 7663, 1980.

Brecht, S. H., Hybrid simulations of the magnetic topology of Mars, J. Geophys. Res., 102, 4743, 1997.

Chen, R. H., T. E. Cravens, and A. F. Nagy, The Martian ionosphere in light of the Viking observations, J. Geophys. Res., 83, 3871, 1978.
DeZeeuw, D. L., et al., A parallel adaptive high-resolution scheme for MHD with applications in space plasma physics, SIAM J. Scient. Comput., submitted, 1999.

Fox, J. L., The production and escape of nitrogen atoms on Mars, J. Geophys. Res., 98, 3297, 1993.

Grard, R., A. Pedersen, S. Klomov, S. Savin, A. Skalsky, J. G. Trotignon, and C. Kennel, First measurements of plasma waves near Mars, Nature, 341, 607, 1989.

Groth, C. P. T., et al., A parallel solution-adaptive scheme for ideal magnetohydrodynamics, AIAA Paper 99-3273, June, 1999.

Hanson, W. B., S. Sanatini, and D. R. Zuccaro, The Martian ionosphere as observed by the Viking retarding potential analyzer, J. Geophys. Res., 82, 4351, 1977.

Lundin, R., et al., First measurements of the ionospheric plasma escape from Mars, Nature, 341, 609, 1989.

Miller, K. L., and R. C. Whitten, Ion dynamics in the Venus ionosphere, Space Sci. Rev., 55, 165, 1991.

Murawski, K., and R. S. Steinolfson, Numerical simulations of mass loading in the solar wind interaction with Venus, J. Geophys. Res., 101, 2547, 1996.

Powell, K. G., et al., A solution-adaptive upwind scheme for ideal magnetohydrodynamics, J. Comp. Phys., submitted, 1999.

Schunk, R. W., and A. F. Nagy, in Ionospheres, Cambridge University Press, in press, 1999.

Shinagawa, H., and S. W. Bougher, A two-dimensional MHD model of the solar wind interaction with Mars, Earth Planets Space, 51, 55, 1999.

Slavin, J. A., et al., The solar wind interaction with Venus: Pioneer Venus observations of bow shock location and structure, J. Geophys. Res., 85, 7625, 1980.

Tanaka, T., Effects of decreasing ionospheric pressure on the solar wind interaction with non-magnetized planets, Earth Planets Space, 50, 259, 1998.

Tanaka, T., and K. Murawski, Three-dimensional MHD simulation of the solar wind interaction with the ionosphere of Venus: Results of two-component reacting plasma simulation, J. Geophys. Res., 102, 19,805, 1997.

Taylor, H. A., et al., Global observations of the composition and dynamics of the ionosphere of Venus: Implications for the solar wind interaction, J. Geophys. Res., 85, 7765, 1980.

Trotignon, J. G., et al., Martian planetopause as seen by the plasma wave system onboard Phobos $2, J$. Geophys. Res., 101, 24,965, 1996.

Vignes, D., et al., Determination of the Martian bow shock and the magnetic pile-up boundary by the MAG/ER experiment onboard Mars Global Surveyor, Geophys. Res. Lett., submitted, 1999.

Zhang, M. H. G., J. G. Luhmann, and A. J. Kliore, An observational study of the nightside ionospheres of Mars and Venus with radio occultation methods, J. Geophys. Res., 95, 17,095, 1990.

Darren L. DeZeeuw, Tamas I. Gombosi, Clinton P. T. Groth, Yifan Liu, Andrew F. Nagy, Space Physics Research Laboratory, Department of Atmospheric, Oceanic, and Space Sciences, University of Michigan, Ann Arbor, MI 48109. (e-mail: liuyifan@engin.umich.edu)

Kenneth G. Powell, Aerospace Engineering Department, University of Michigan, Ann Arbor, MI 48109. (e-mail: powell@engin.umich.edu)

(Received April 12, 1999; revised June 24, 1999; accepted June 28,1999 .) 